\title{
Smurf2 up-regulation activates telomere-dependent senescence
}

\author{
Hong Zhang ${ }^{1}$ and Stanley N. Cohen ${ }^{1,2,3}$ \\ ${ }^{1}$ Department of Genetics and ${ }^{2}$ Department of Medicine, Stanford University School of Medicine, \\ Stanford, California 94305-5120, USA
}

\begin{abstract}
Progressive telomere shortening activates replicative senescence, which prevents somatic cells from being propagated indefinitely in culture. The limitation of proliferative capacity imposed by replicative senescence is thought to contribute to both organismal aging and the prevention of tumor development. Here we report that up-regulation of Smurf2, an E3 ubiquitin ligase previously implicated in TGF- $\beta$ signaling, is a specific consequence of telomere attrition in human fibroblasts and that such up-regulation is sufficient to produce the senescence phenotype. Adventitious production of the Smurf2 protein in early passage fibroblasts at the same physiological level observed during telomere-mediated senescence resulted in proliferative arrest in a viable state, morphological and biochemical alterations characteristic of senescence, acquisition of senescence-specific alterations in gene expression, and reversal of cellular immortalization by telomerase. We show that the senescence-inducing actions of Smurf2 occur in the absence of detectable DNA damage or stress response, that Smurf2's effects require a novel function distinct from its E3 activity, that Smurf2 recruits the $\mathrm{Rb}$ and $\mathrm{p} 53$ pathways for senescence induction, and that while p21 is elevated by Smurf2, Smurf2-mediated senescence is independent of p21. Smurf2 is the first gene found to be both up-regulated by telomere attrition and sufficient to induce senescence.
\end{abstract}

[Keywords: Senescence; telomere; Smurf2; p53; Rb]

Supplemental material is available at http://www.genesdev.org.

Received August 23, 2004; revised version accepted October 15, 2004.

Populations of human somatic cells cannot divide indefinitely in culture, and at the end of their replicative life span, enter an irreversible growth arrest state termed replicative senescence (Hayflick and Moorhead 1961). While a role for this limitation of proliferative capacity in organismal aging remains uncertain, increasing evidence suggests that replicative senescence is a tumorsuppression mechanism that impedes the accumulation of mutations required for neoplastic transformation (for reviews, see Wright and Shay 2001; Kim et al. 2002). The onset of replicative senescence is determined by a mitotic clock that records the number of cell divisions, and the progressive shortening of chromosomal telomeres during each DNA replication cycle is believed to calibrate this clock (Hayflick 1976; Harley 1991; Holt et al. 1996; Blackburn 2001; Wright and Shay 2002). Adventitious expression of the catalytic subunit of telomerase (hTERT), which normally is not produced in human somatic cells (Kim et al. 1994), can prevent such telomere shortening and abrogate senescence-related growth arrest (Bodnar et al. 1998; Kiyono et al. 1998; Vaziri and Benchimol 1998).

${ }^{3}$ Corresponding author.

E-MAIL sncohen@stanford.edu; FAX (650) 725-1536.

Article published online ahead of print. Article and publication date are at http://www.genesdev.org/cgi/doi/10.1101/gad.1253004.
The ability of telomere length to modulate replicative senescence distinguishes this state mechanistically from telomere-independent arrest of cell proliferation (Bodnar et al. 1998; Wright and Shay 2001). The mechanisms proposed to account for the effects of telomere shortening include induction of a DNA damage response by the uncapping of DNA ends or the alteration of telomere structure (Blackburn 2001; Karlseder et al. 2002; d'Adda di Fagagna et al. 2003; Stewart et al. 2003; Takai et al. 2003), and activation of ordinarily silent human "senescence genes" located in subtelomeric regions (Wright and Shay 1992).

We recently identified a gene expression signature that is unique to telomere-dependent senescence in human fibroblasts and thus distinguishes the senescence phenotype from other processes affecting cell cycling and growth arrest (Zhang et al. 2003). Among the differentially expressed genes in this signature is Smurf2, which encodes an E3 ubiquitin protein ligase involved in Smadmediated TGF- $\beta$ signaling (Kavsak et al. 2000; Lin et al. 2000; Zhang et al. 2001). Here we show that up-regulation of Smurf2 is a specific consequence of telomere shortening in human fibroblasts and that such up-regulation is sufficient to produce the senescence phenotype. Adventitious expression of Smurf2 in early passage fibroblasts to the level normally found in senescent cells re- 
sulted in proliferative arrest in a viable state, morphological and biochemical alterations characteristic of senescence, acquisition of senescence-specific alterations in gene expression, and reversal of cellular immortalization by hTERT. Induction of senescence by Smurf2 was prevented by concurrent expression of the E6 and E7 oncoproteins of human papillomavirus 16 (HPV16), but not by either protein alone, indicating Smurf2's use of the $\mathrm{Rb}$ and p53 pathways to produce the senescence phenotype.

\section{Results}

Up-regulation of Smurf2 is induced by telomere attrition during replicative senescence in human fibroblasts

The HECT family E3 ubiquitin protein ligase Smurf2 is implicated in the ubiquitination and down-regulation of Smad1, Smad2, type I TGF- $\beta$ receptor, and the transcription corepressor SnoN (Kavsak et al. 2000; Lin et al. 2000; Bonni et al. 2001; Zhang et al. 2001). Whereas TGF- $\beta$ inhibits proliferation of cells of epithelial origin, it can stimulate proliferation of fibroblasts and other mesenchymal cells (Massague 2000). Thus, our observation that Smurf2 mRNA was elevated during replicative senescence of human fibroblasts (Zhang et al. 2003) prompted further study.

Northern blot analysis using Smurf2 cDNA as the probe identified hybridizing transcript species $\sim 3.0 \mathrm{~kb}$ and $\sim 6.0 \mathrm{~kb}$ in length. Whereas senescent fibroblasts showed up-regulation of both transcript species (Fig. 1A, WS1 and WI38), the Smurf2 level remained constant in populations of human mammary epithelial cells (HMECs) (Fig. 1A, 48R and 184) during their progression to replicative senescence-consistent with earlier evidence that senescence occurs by fundamentally different pathways in these two kinds of cells (Romanov et al. 2001; Zhang et al. 2003, 2004). Consistent with our Northern blot results, two isoforms of a protein recognized by anti-Smurf2 antibody were elevated three- to sixfold during replicative senescence in fibroblasts (Fig. 1B). No elevation of Smurf 2 transcription or protein synthesis was observed in quiescent populations of any of the fibroblast cell lines we examined (Fig. 1A,B), indicating that up-regulation of Smurf 2 is not a general concomitant of proliferative arrest. Importantly, fibroblasts immortalized by adventitious expression of hTERT and analyzed after multiple passages in culture showed no increase in Smurf2 expression (Fig. 1C), indicating that up-regulation of Smurf 2 is not the result of extended cell passage per se but, rather, is a consequence of telomere shortening. Expression of the closely related gene, Smurf1, was not affected during senescence in any of the cell lines tested (data not shown).

\section{Smurf2 induction is not an unspecific concomitant of cellular stress or DNA damage}

Exposure to oxidative and certain other stress conditions commonly results in a state of proliferative arrest that

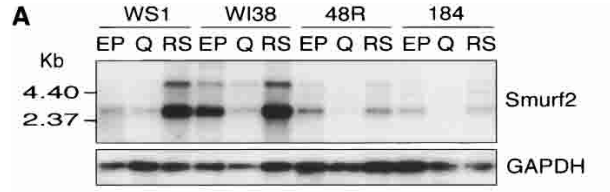

$\mathbf{B}$

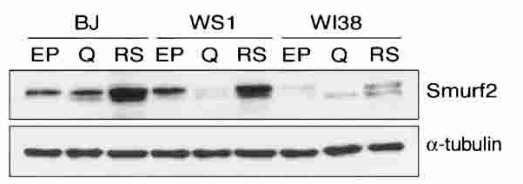

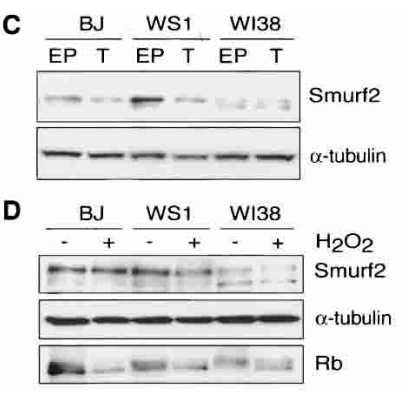

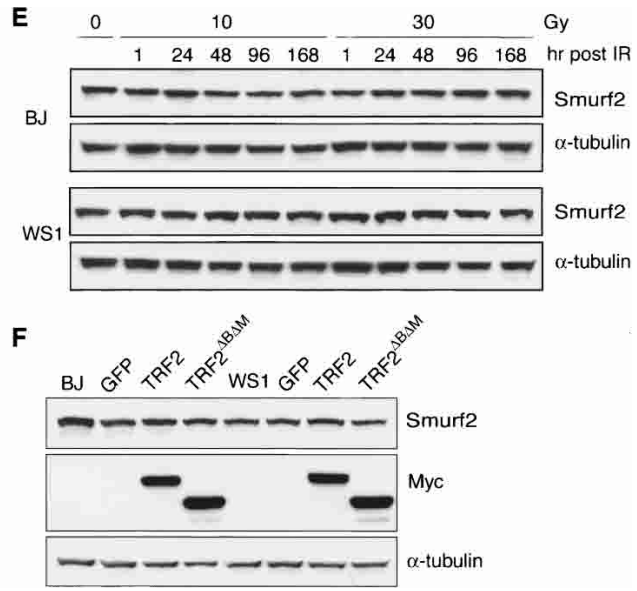

Figure 1. Increased expression of Smurf2 during replicative senescence in human fibroblasts. (A) Northern analysis of Smurf2 expression in cells that are early passage proliferating (EP), quiescent (Q) by contact inhibition, and replicative senescent (RS) by serial passage. The probe used was the cDNA of Smurf2 and GAPDH as loading control. Two fibroblast lines (WS1 and WI38) and two HMEC lines (48R and 184) were analyzed. $(B)$ Western blot analysis of Smurf2 protein level in human fibroblasts that are early passage proliferating (EP), quiescent (Q), or senescent (RS). Three fibroblast lines (BJ, WS1, and WI38) were used. $\alpha$-Tubulin was used as loading control. $(C)$ Expression of hTERT and consequent immortalization prevents the induction of Smurf2. Western blot analysis of the Smurf2 protein level in human fibroblasts (BJ and WS1) of early passage (EP) and hTERT-immortalized (T) cells. $(D)$ Expression of Smurf2 is not induced by oxidative stress. Western blot analysis in early passage fibroblasts treated with a sublethal dose of $\mathrm{H}_{2} \mathrm{O}_{2}(250$ $\mu M)$. (E) DNA damage response activated by ionizing radiation does not induce Smurf2. Early passage BJ and WS1 were irradiated with different doses, and cells were analyzed 1, 24, 48, 96, or $168 \mathrm{~h}$ after irradiation using Western blotting. (F) Expression of Smurf2 protein is not elevated by the expression of the dominant-negative allele of TRF2 (TRF2 ${ }^{\Delta \mathrm{B} \Delta \mathrm{M}}$ ). Early passage BJ and WS1 fibroblasts were infected with retrovirus to express Myc-tagged TRF2 or TRF2 ${ }^{\Delta \mathrm{B} \Delta \mathrm{M}}$, or GFP control, selected with puromycin resistance for a week, and analyzed using Western blotting. 
also has been termed "senescence." While exposure to stress is accompanied by some of the same biochemical changes that occur during senescence induced by telomere shortening, for example, decreased phosphorylation of $\mathrm{Rb}$ and positive staining for $\beta$-galactosidase activity at pH 6 (Di Leonardo et al. 1994; Chen et al. 1995; von Zglinicki et al. 1995; Serrano et al. 1997; Robles and Adami 1998), stress-induced proliferative arrest is not associated with telomere attrition and is not abrogated by adventitious expression of telomerase (Wei and Sedivy 1999; Gorbunova et al. 2002). Consistent with the conclusion that Smurf2 up-regulation is a specific consequence of telomere shortening, we found that the steady-state level of Smurf2 was unaffected by treatment with $\mathrm{H}_{2} \mathrm{O}_{2}$ (Fig. 1D), which induces a senescence-like stress response as indicated by decreased phosphorylation of $\mathrm{Rb}$ (Fig. 1D) and positive staining for $\beta$-galactosidase activity at $\mathrm{pH} 6$ (data not shown).

The cessation of cell proliferation associated with telomere shortening is proposed to be a response to DNA damage produced by uncapping or structural alteration of chromosome ends (Blackburn 2001; Karlseder et al. 2002; d'Adda di Fagagna et al. 2003; Takai et al. 2003), and recently, evidence for activation of a DNA damage response by dysfunctional telomeres has been obtained (d'Adda di Fagagna et al. 2003; Takai et al. 2003; Herbig et al. 2004). We carried out two types of experiments to learn whether DNA damage either generally within cells, or specifically at telomeres, reproduced the upregulation of Smurf2 expression we observed in senescent fibroblasts undergoing gradual telomere attrition: firstly, we generated double-strand DNA breaks in early passage BJ and WS1 cells by treating the cells with ionizing radiation. As expected, a typical DNA damage response-as indicated by the appearance of nuclear foci of phosphorylated histone H2A.X ( $\gamma$-H2A.X) was observed within $1 \mathrm{~h}$ after irradiation (Fig. 3, below), and entry into a senescence-like growth arrest state occurred after 4-7 d (Di Leonardo et al. 1994). However, despite a much larger number of nuclear $\gamma$-H2A.X foci in populations of irradiated cells than was observed in cells undergoing replicative senescence due to telomere attrition (Fig. 3, below), irradiated cells showed no change in Smurf2 level (Fig. 1E), supporting the argument that up-regulation of Smurf2 is not a general concomitant of the DNA damage response. Similar results were obtained following treatment of early passage fibroblasts with the DNA-damaging agents Adriamycin or etoposide (data not shown).

In a second set of experiments, we adventitiously expressed $T R F 2^{\triangle \mathrm{B} \Delta M}$, a dominant-negative allele of the telomere-binding protein TRF2 in early passage fibroblasts. Recent investigations have shown that expression of $\mathrm{TRF} 2^{\Delta \mathrm{B} \Delta \mathrm{M}}$ results in massive deprotection of telomeres (van Steensel et al. 1998; de Lange 2002) and consequent activation of the cellular response to DNA damage (Takai et al. 2003). While these acute effects of $\mathrm{TRF}^{\Delta \mathrm{B} \Delta \mathrm{M}}$ do not exactly mimic the gradual telomere attrition that occurs during replicative senescence (Herbig et al. 2004), expression of TRF $2^{\Delta \mathrm{B} \Delta \mathrm{M}}$ in early passage human fibroblasts nevertheless produces a senescence- like phenotype in the absence of telomere shortening (Smogorzewska and de Lange 2002). We found that expression of Smurf 2 was unchanged following TRF2 ${ }^{\Delta \mathrm{B} \Delta \mathrm{M}}$ expression in BJ and WS1 fibroblasts (Fig. 1F), arguing that telomere damage and dysfunction per se is not sufficient to induce Smurf2 expression, but, rather, that Smurf2 up-regulation is a specific response to the telomere attrition that occurs physiologically as cells enter senescence.

\section{Adventitious expression of Smurf2 results in senescence of early passage human fibroblasts}

Is up-regulation of Smurf2 during telomere attrition sufficient to produce the senescence phenotype? To address this question, we investigated the effects of producing Smurf2 adventitiously in three separate lines of early passage human fibroblasts to the same level observed normally during telomere-mediated senescence (Fig. 2). Full-length Smurf2 cDNA was expressed in BJ, WS1, and WI38 cells using the retrovirus-based vector pBabeSmurf2-IRES-Puro; an identical construct expressing the jellyfish green fluorescent protein (GFP) was used as control. After growth in the presence of puromycin, virtually every puromycin-resistant cell in control populations was found microscopically to express GFP /data not shown), indicating the effectiveness of the IRES for expression of the tandemly positioned genes. The Smurf2infected early passage cell populations showed a three- to sixfold increase in abundance of Smurf2 protein versus GFP-infected control cells (Fig. 2A), and had a Smurf2 steady-state level quantitatively similar to the level occurring physiologically in fibroblasts undergoing replicative senescence after extended passage in culture (cf. Fig. 1B).

Whereas control cell populations infected with pBabeGFP-IRES-Puro grew exponentially until the cells reached confluence, adventitious expression of Smurf2 to the level observed physiologically during replicative senescence resulted in arrest of proliferation of all three lines of early passage fibroblasts in a subconfluent state (Fig. 2B). The early passage cells in which Smurf2 was adventitiously elevated failed to proliferate in the presence of $10 \%$ serum but instead remained viable in a state of replicative arrest during more than a month of observation. Serial examination by light microscopy (Fig. 2C) showed that the Smurf2-arrested early passage cells were enlarged and had undergone an acute transition-within $6 \mathrm{~d}$ of infection by the Smurf2-expressing virus-to the flat morphology characteristic of cells entering senescence at the end of their replicative life span. Additionally, during this acute transition, the early passage Smurf2-expressing cells acquired the property of positive staining for $\beta$-galactosidase activity at $\mathrm{pH} 6$ (Fig. 2D), which commonly, albeit not specifically, is associated with the replicative senescence phenotype (Dimri et al. 1995; Robles and Adami 1998; Severino et al. 2000; Ramirez et al. 2001). The induction of senescence by Smurf2 was not accompanied by any evidence of DNA damage response, as assessed by the occurrence of 
Figure 2. Adventitious expression of Smurf2-induced senescence in early passage human fibroblasts (BJ, WS1, and WI38). (A) Western blot analysis of Smurf2 protein in early passage cells infected with retrovirus expressing GFP, Smurf2, or ligase mutant C716A and selected for puromycin resistance. (B) Proliferative potentials of early passage cells infected with retrovirus containing GFP, Smurf2, or ligase mutant C716A. After selection for puromycin resistance, infected cells were plated at low density and cell numbers were counted daily. A represented growth curve for each cell line was shown. (C) Crystal violet staining of early passage cells after infection with retrovirus containing GFP, Smurf2, or ligase mutant C716A. (D) Staining for $\beta$-galactosidase activity at $\mathrm{pH} 6$ of early passage cells after infection with retrovirus containing GFP, Smurf2, or ligase mutant C716A.
A

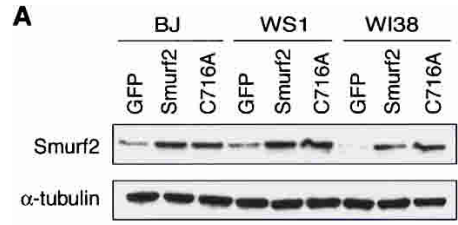

B
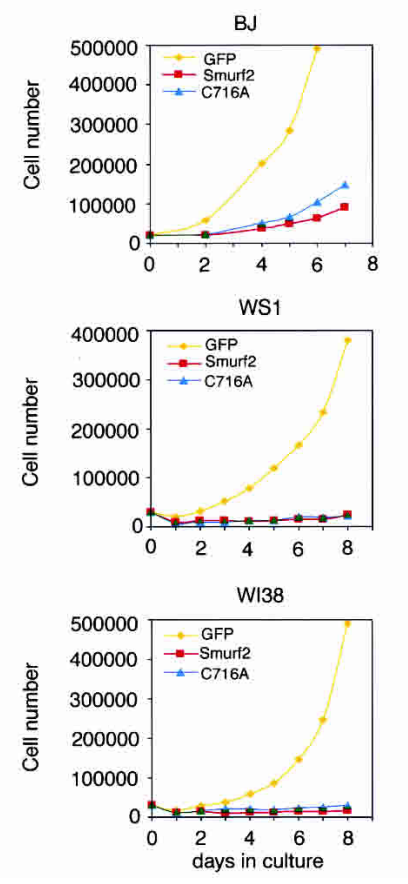
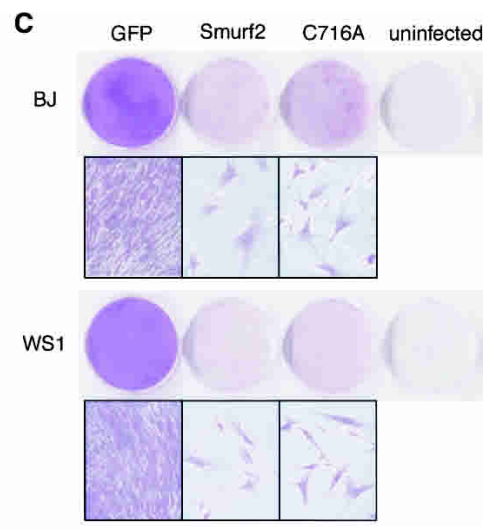

WI38

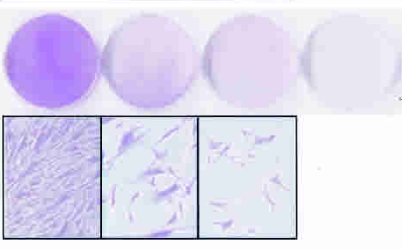

D

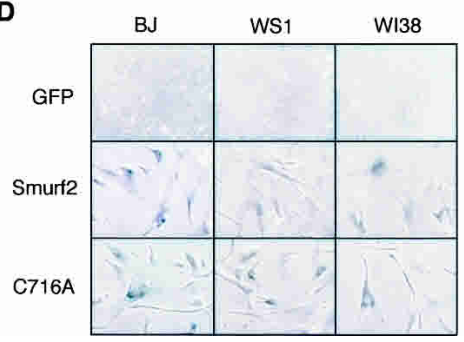

nuclear $\gamma$-H2A.X loci in either BJ cells (Fig. 3) or WS1 cells (data not shown).

Microarray analysis of gene expression provided further evidence that up-regulation of Smurf2 in early passage fibroblasts results in the rapid acquisition by these cells of properties characteristic of replicative senescence. Using human cDNA microarrays, we globally compared gene expression in early passage cells producing Smurf2 adventitiously with gene expression in a GFP-expressing early passage control cell population. As shown in Figure 4A, we found that the gene expression signature associated with adventitious expression of Smurf2 showed 194 up-regulated and 177 down-regulated genes (Supplementary Table S1) in common with the signature observed in cells undergoing replicative senescence as a consequence of telomere shortening (Zhang et al. 2003). Additional transcriptional alterations (142 up-regulated and 206 down-regulated genes) (Supplementary Table S2) were observed during telomere-mediated-but not Smurf2-mediated-senescence (Fig. 4B), possibly identifying loci that act prior to Smurf2 activation in the senescence program. In contrast, the profile of genes whose expression was altered during adventitious production of Smurf2 in early passage fibroblasts showed limited commonality (17 upregulated and five down-regulated) with the quiescencespecific gene expression profile reported previously (Zhang et al. 2003). Adventitious expression of Smurf2 protein in early passage cells also resulted in altered expression of some genes (79 up-regulated and 19 downregulated) (Supplementary Table S3) that were not affected by telomere attrition (Fig. 4C); these alterations may reflect Smurf 2 actions unassociated with its role in senescence and/or differences in the physiological state of cells before and after repeated passage in culture.

\section{Smurf2 expression produces senescence in hTERT-immortalized cells}

Expression of hTERT in primary cultures of human fibroblasts precludes the progressive shortening of telomeres that activates events leading to senescence, resulting in the immortalization of cell populations (Bodnar et al. 1998; Vaziri and Benchimol 1998; Dickson et al. 2000). In strong support of the conclusion that Smurf2 expression is sufficient to produce the senescence phenotype, we found that adventitious expression of Smurf2 to the level normally observed during replicative senescence induced by telomere attrition (cf. Figs. 5A and 1B) reversed hTERT-mediated immortalization of human fibroblasts. In these experiments, human fibroblast cell lines (BJ-hTERT and WS1-hTERT), whose immortalization by hTERT had been demonstrated by passage for $>150$ cell population doublings (more than twice the number of their nonimmortalized parental cells; data not shown), were infected with pBabe-Smurf2-IRES-Puro 


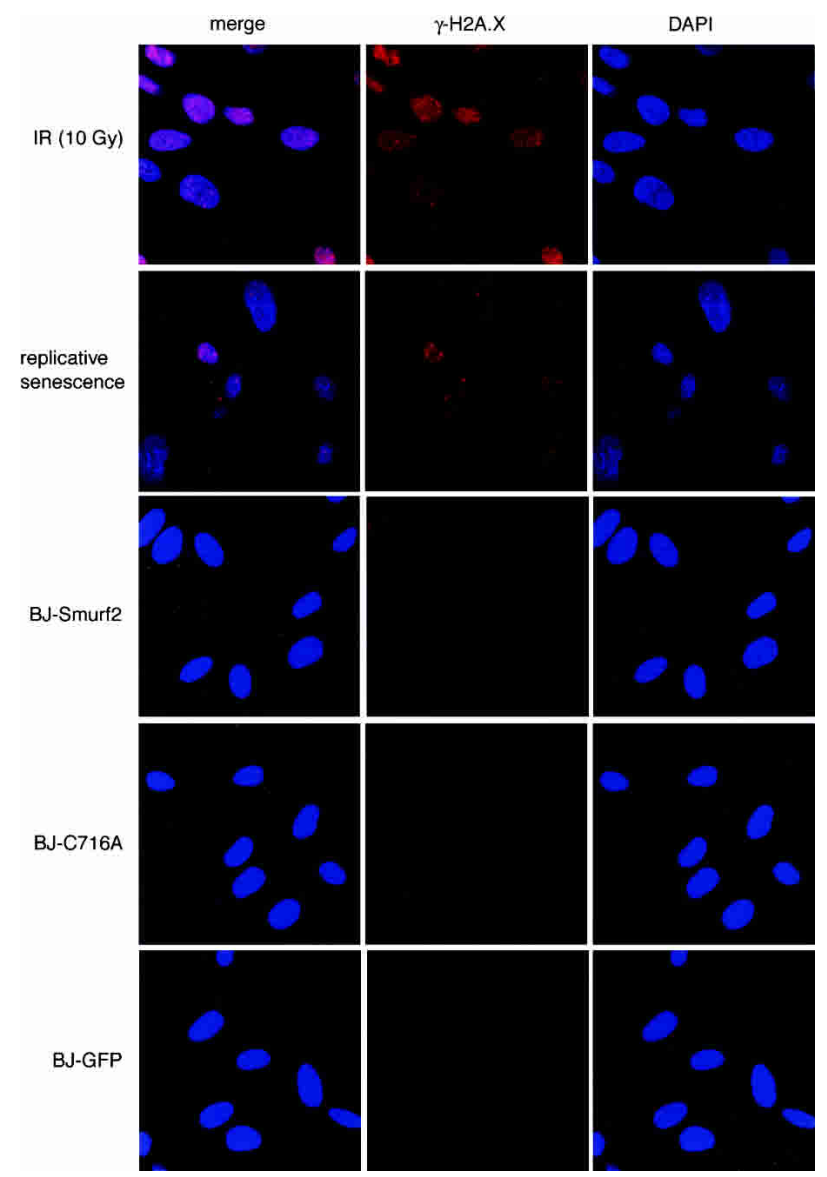

Figure 3. Lack of DNA damage response in Smurf2-induced senescent cells. BJ cells of replicative senescence, Smurf2-induced senescence, and GFP expressing control, as well as early passage cells $1 \mathrm{~h}$ after being treated with $10 \mathrm{~Gy}$ of ionizing radiation (IR), were stained with anti-phospho-histone H2A.X (Ser 139) antibody and DAPI.

retrovirus expressing Smurf2. As had been observed for early passage human fibroblasts, adventitious expression of Smurf2 in hTERT-immortalized fibroblasts resulted in the cessation of cell proliferation in a subconfluent state of growth (Fig. 5B), an enlarged and flat cell morphology, and positive staining for $\beta$-galactosidase activity at $\mathrm{pH} 6$ (Fig. 5C).

The senescence-inducing function of Smurf2 is independent of its ubiquitin protein ligase activity

The only previously known function attributed to Smurf2 is its action as a ubiquitin ligase (E3 enzyme) involved in Smad-mediated TGF- $\beta$ signaling. Earlier work has shown that the conserved Cys residue at position 716 is required for this function (Kavsak et al. 2000; Lin et al. 2000; Zhang et al. 2001). To test whether the senescence-inducing ability of Smurf 2 is dependent on Smurf2's E3 enzyme activity, we introduced mutations that changed Cys 716 to Ala. Adventitious expression of the resulting Smurf2 mutant protein (C716A) induced a senescence phenotype in human fibroblasts that was indistinguishable from the phenotype induced by the wildtype Smurf2 protein (Figs. 2-5). Moreover, the expression profile for genes reported to be regulated by TGF- $\beta$ in human epithelial cells (Kang et al. 2003) was not recapitulated in early passage fibroblasts that adventitiously express Smurf2 or by up-regulation of Smurf2 during replicative senescence induced by telomere shortening (Supplementary Fig. S1). Collectively, these findings support the argument that the senescence-producing effects of Smurf 2 are not mediated by its actions as a ubiquitin ligase and consequently not by TGF- $\beta$ signaling.

During the cloning of Smurf2 cDNA, we identified a form of Smurf 2 cDNA lacking a 39-bp region that corresponds to exon 2 of the gene and encodes a 13-amino acid region that is part of the phospholipid/calcium-binding C2 domain. This shorter form of Smurf2, which has been

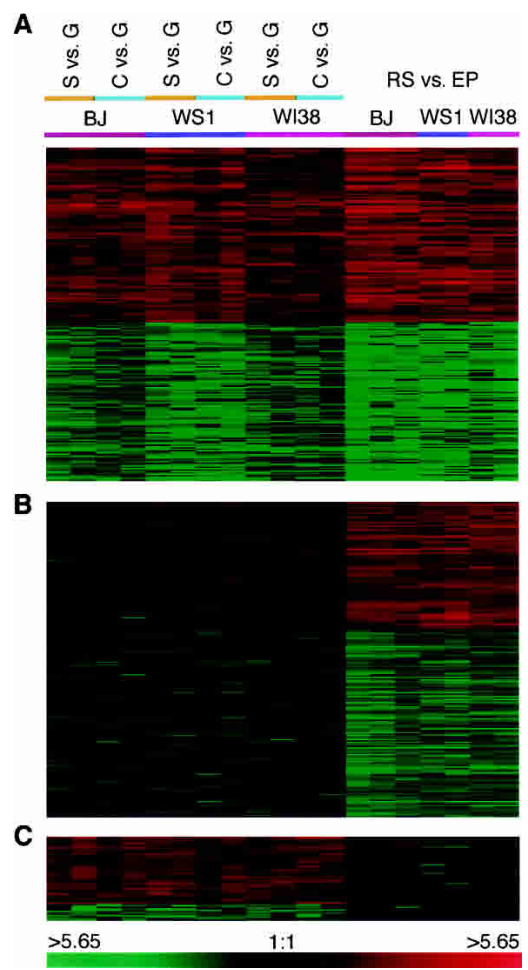

Figure 4. Microarray analysis of gene expression alterations in Smurf2-induced senescent cells. Transcripts from senescent cells induced by Smurf2 or its ligase mutant C716A were labeled with Cy5-dUTP (red), and hybridized onto microarrays with Cy3-dUTP (green)-labeled transcripts from GFP-expressing control cells. Data for replicative senescence in fibroblasts were retrieved from a previously published study (Zhang et al. 2003). In that data set, transcripts from replicative senescence (labeled with Cy5-dUTP) were compared with those from early passage proliferating cells (labeled with Cy3-dUTP). Data were analyzed using GABRIEL as described in Materials and Methods. $(A)$ Transcriptional alterations in common between Smurf2-induced senescence and telomere-mediated replicative senescence. $(B)$ Transcriptional alterations occurring only during replicative senescence but not during Smurf2-induced senescence. (C) Transcriptional alterations induced by Smurf2 elevation only. 
A

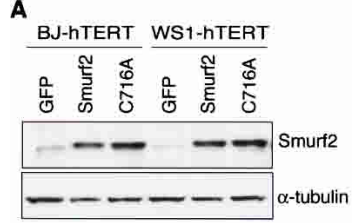

B
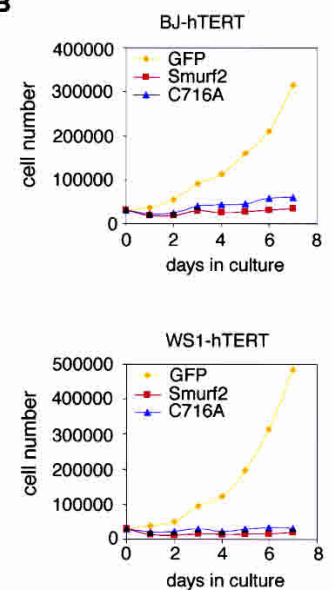

Figure 5. Elevation of Smurf2 reversed cellular immortalization by hTERT. GFP, Smurf2, and C716A were adventitiously expressed in hTERT-immortalized human fibroblast cell lines (BJ-hTERT and WS1-hTERT) and selected for puromycin resistance. $(A)$ Western blot analysis of Smurf2 protein expression. (B) Proliferative potential of these cells was monitored as described in Materials and Methods. $(C)$ Crystal violet and $\mathrm{pH} 6$ $\beta$-galactosidase activity staining.

identified as an alternatively spliced variant, likely comprises the low-molecular-weight band in Western blotting (Fig. 1). Expression of this cDNA (Smurf2-AS) in early passage fibroblasts also produced the senescence phenotype (data not shown), indicating that the C2 domain is not required. To identify the Smurf 2 regions that are responsible for the protein's senescence-inducing ability, cDNAs encoding various domains were separately generated by PCR. In control experiments, the ability of the resulting retrovirus-based Smurf 2 mutants to infect cells and be expressed was confirmed in a human fibrosarcoma cell line (HT1080) that is not responsive to senescence induction (Fig. 6), indicating that all of the constructs are functional. Additionally, after $4 \mathrm{~d}$ of growth under selection for puromycin, similar numbers of cells were observed for all of the Smurf 2 mutants (data not shown). As seen in Figure 6, a combination of three WW protein-interaction domains present on Smurf2 (Kavsak et al. 2000; Lin et al. 2000; Zhang et al. 2001) with its HECT domain (i.e., $\Delta$ C2) caused proliferative arrest in early passage fibroblasts WS1 and WI38, whereas expression of these domains individually or of the separate C2 domain had no effect. Moreover, while the first WW domain located between amino acids 149 and 242 was necessary for the induction of senescence by Smurf2, it is not required for Smurf2's interaction with Smads (Kavsak et al. 2000; Lin et al. 2000; Zhang et al. 2001). p21 function is not required for Smurf2-induced senescence

In a search for genes acting downstream of Smurf2 in the telomere-dependent senescence pathway, we found that adventitious expression of Smurf2 resulted in the elevation of p21 and cyclin D1 (Fig. 7B,C), both of which are known to be up-regulated during replicative senescence associated with telomere shortening (Dulic et al. 1993; Noda et al. 1994; Atadja et al. 1995; Tahara et al. 1995; Stein et al. 1999; see also Fig. 7A), but not during quiescence (Fig. 7A). In contrast to p21 and cyclin D1, p27 and cyclin D2, members of their respective families, were unchanged during senescence (Fig. 7; data not shown). Concurrent up-regulation of both $\mathrm{p} 21$ and cyclin D1 during either telomere-mediated or Smurf2-induced senescence is paradoxical, as these proteins have contrary actions on the activation of cyclin-dependent kinases (CDKs), which phosphorylate Rb (Sherr 1994; Sherr and Roberts 1999). In our microarray analysis, we observed that genes identified in a previous DNA microarray study as cyclin D1 targets (Lamb et al. 2003) did not show altered expression in cells induced to enter senescence by either Smurf2 or telomere shortening (Supplementary Fig. S2), suggesting that the elevation of cyclin D1 observed during senescence does not have its usual downstream consequence. Consistent with this conclusion, we found that phosphorylation of $\mathrm{Rb}$, which ordinarily is increased in cells overexpressing cyclin D1 (Sherr 1994), was decreased by Smurf2, as well as by telomere-mediated senescence (Fig. 7). In contrast to the absence of detectable effects of cyclin D1 elevation, genes normally up- or down-regulated by p21 /Chang et al. 2000) showed the expected change in expression during both Smurf2-induced and telomere-mediated senescence (Supplementary Figs. S3, S4).

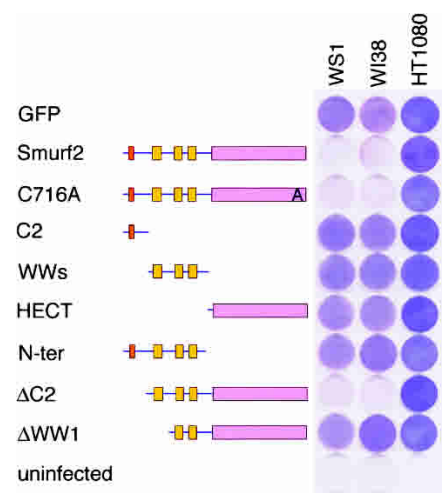

Figure 6. Domains of Smurf2 that are necessary for its senescence-inducing function. Different portions of Smurf2, including the C2 domain (amino acids 1-147), three WW domains (amino acids 149-344), the HECT domain (amino acids 345748), N-ter (amino acids 1-344), $\Delta \mathrm{C} 2$ (amino acids 149-748), and $\triangle$ WW1 (amino acids 242-748), were cloned into pBabe-IRESPuro using cDNA generated by PCR, and ectopically expressed in two early passage fibroblast lines (WS1 and WI38) and a human fibrosarcoma cell line HT1080. After selection with puromycin, plates were stained with crystal violet. 
We used $\mathrm{p} 21^{-/-}$cells generated through two sequential rounds of homologous recombination (Brown et al. 1997) to learn whether the senescence phenotype induced by Smurf2 requires $\mathrm{p} 21$ function, and found that it does not. Adventitious expression of Smurf2 (Fig. 8A) promptly induced cessation of proliferation in a subconfluent state of growth in cultures of $\mathrm{p} 21^{-/-}$fibroblasts, their $\mathrm{p} 21^{+/+}$ parental LF1 cells (data not shown), and $\mathrm{p}^{1^{-/-}}$or $\mathrm{p} 21^{+/+}$ LF1 cells that we had immortalized by introduction of an hTERT-expressing construct (Fig. 8B). Proliferative arrest in these cell lines was associated with an enlarged and flat cell morphology and positive staining for $\beta$-galactosidase activity at pH 6 (Fig. 8C). As occurs during replicative senescence induced by telomere attrition, expression of cyclin D1 remained elevated in p21-null mutant cells induced to enter replicative senescence by adventitious expression of Smurf2 (Fig. 8A).

\section{Smurf2-induced senescence requires a functional p53 or $R b$ pathway}

Replicative senescence of human cells secondary to telomere shortening can be abrogated by concurrent suppres-

A

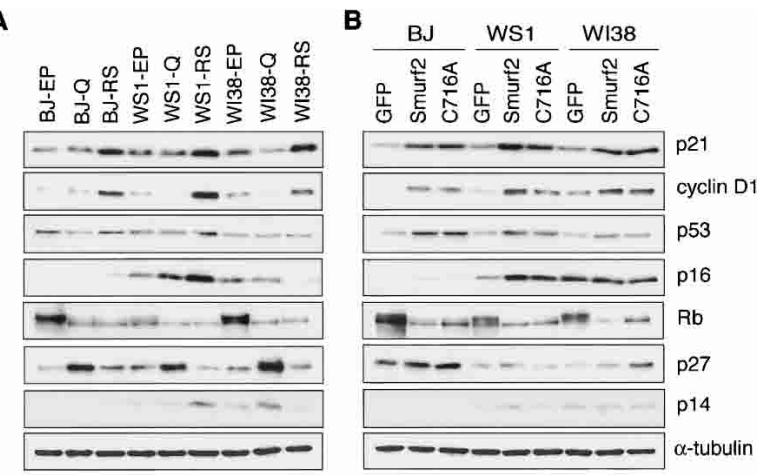

C

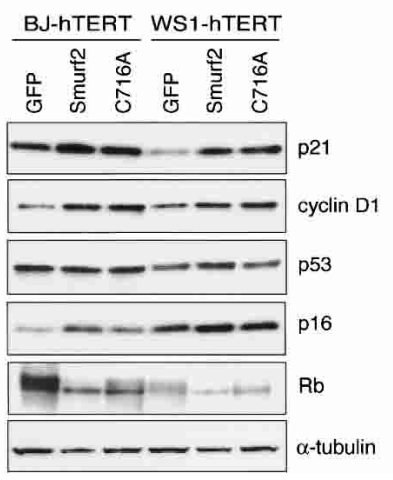

Figure 7. Concurrent elevation of $\mathrm{p} 21$ and cyclin D1 during Smurf2-induced senescence as well as during replicative senescence. $(A)$ Western blot analysis of various proteins in fibroblasts that are early passage proliferating $(\mathrm{EP})$, quiescent $(\mathrm{Q})$ by contact inhibition, or replicative senescent (RS) by serial passage. $(B)$ Western blot analysis of various proteins in early passage human fibroblasts (BJ, WS1, and WI38) infected with retrovirus expressing control GFP, wild-type Smurf2, or ligase mutant C716A. (C) Protein expression in hTERT-immortalized fibroblasts after adventitiously expressing wild-type Smurf2, ligase mutant C716A, and control GFP.
A

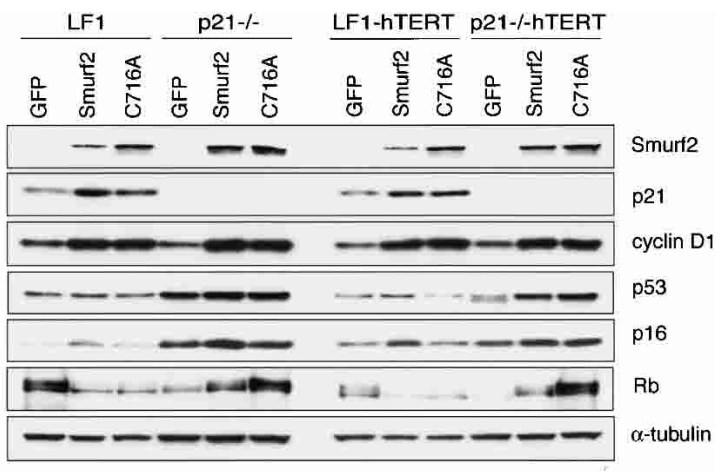

B
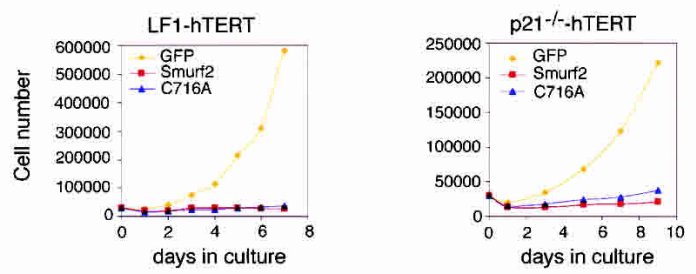

C

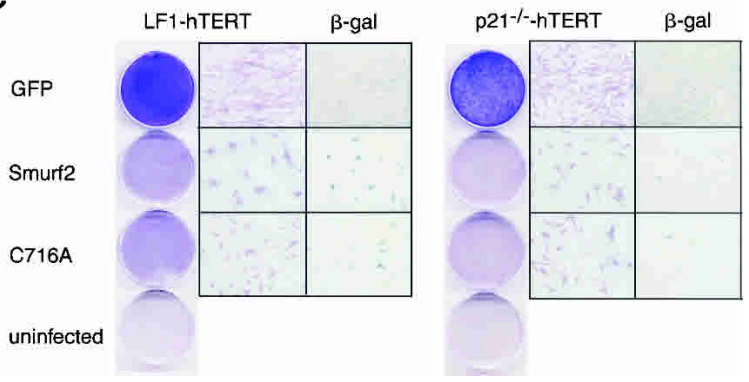

Figure 8. Absence of $\mathrm{p} 21$ does not abrogate the effect of Smurf2 induction. Early passage p $21^{+/+}$LF1 fibroblasts, p $21^{-/-}$fibroblasts, and their hTERT-immortalized derivatives were infected with retrovirus expressing control GFP, wild-type Smurf2, or ligase mutant C716A, and selected with puromycin. (A) Western blot analysis of protein expression in these cells. $(B)$ Growth curves of hTERT-immortalized LF1, p21-null cells after adventitious expression of GFP, Smurf2, and C716A. (C) Crystal violet and $\beta$-galactosidase activity at $\mathrm{pH} 6$ staining in hTERT-immortalized LF1, p21-null cells after adventitious expression of GFP, Smurf2, and C716A.

sion of the p53 and Rb pathways (Shay and Wright 1989; Shay et al. 1991). To test whether the Smurf2-induced senescence also makes uses of these two pathways, we infected early passage BJ cells with lentivirus-expressing HPV16 E6 or E7 oncoproteins to suppress, respectively, the activities of $\mathrm{p} 53$ or $\mathrm{Rb}$ proteins (Munger and Howley 2002), and tested the ability of Smurf2 to induce the senescence phenotype in these cells. Whereas expression of E6 or E7 alone did not prevent Smurf2's effects-as indicated by cell growth arrest in a subconfluent state, enlarged and flat cell morphology, and positive staining for $\beta$-galactosidase activity at $\mathrm{pH} 6$ (Fig. 9) - the concurrent expression of both E6 and E7 proteins abrogated these properties associated with senescence (Fig. 9). These results indicate that a functional p53 or Rb pathway individually is sufficient for Smurf2-induced senes- 
A

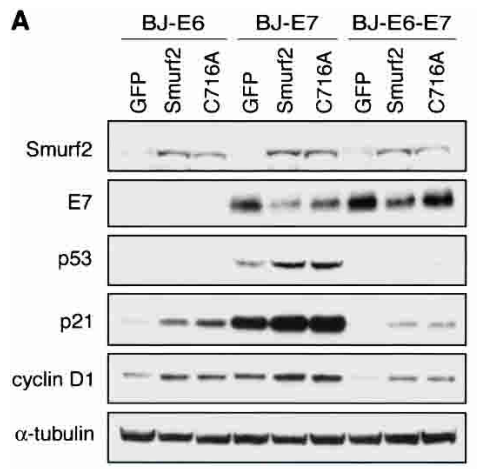

B

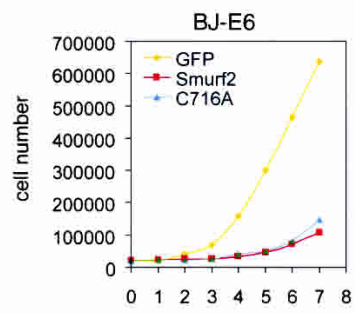

BJ-E7
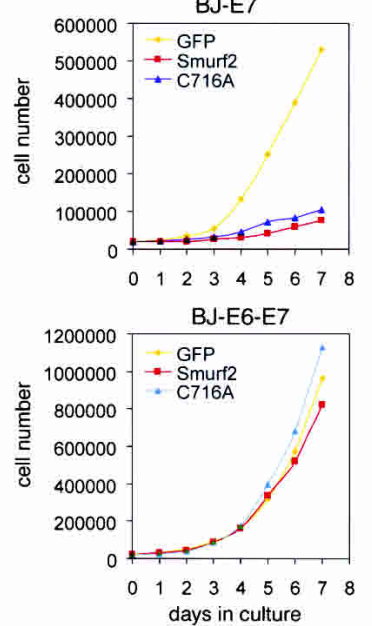

C
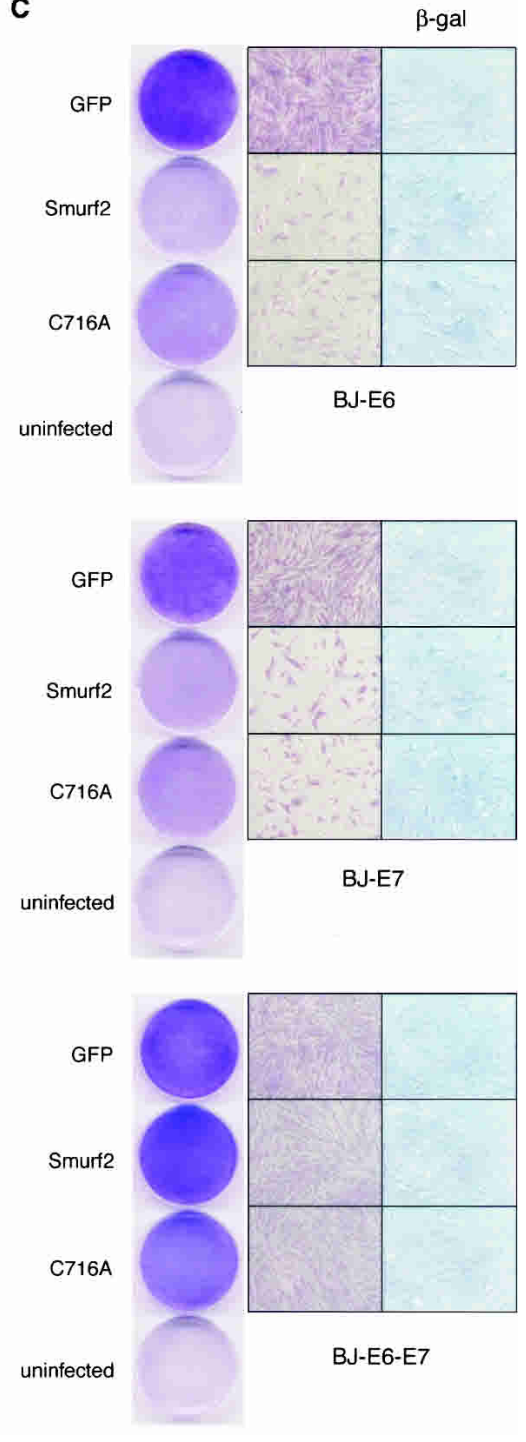

Figure 9. Expression of HPV16 E6 and E7 abrogates Smurf2-induced senescence. Early passage BJ fibroblasts were infected with lentivirus expressing HPV16 E6, E7 alone, or E6 and E7 together. In these E6/E7-expressing populations, Smurf2 as well as GFP control were ectopically expressed using retrovirus. (A) Western blot analysis of protein expression. (B) Growth curves of BJ-E6, BJ-E7, and BJ-E6-E7 populations after adventitious expression of GFP, Smurf2, and C716A. (C) Crystal violet and $\beta$-galactosidase activity at $\mathrm{pH} 6$ staining of BJ-E6, BJ-E7, and BJ-E6-E7 populations after adventitious expression of GFP, Smurf2, and C716A. cence. Interestingly, notwithstanding the expected ability of E6 expression to greatly reduce the steady-state level of p53, and consequently of p53-dependent production of the p21 protein, Smurf2 induction of p21 still occurred in E6-expressing cells (Fig. 9A), supporting the argument that Smurf2 affects p21 expression by a p53independent mechanism.

\section{Discussion}

Replicative senescence in primary cultures of human cells normally is activated by telomere shortening. Our investigations show that the E3 ubiquitin ligase Smurf2 is up-regulated in human fibroblasts undergoing replicative senescence in response to telomere attrition and that telomerase expression in such cells prevents Smurf2 up-regulation. The inability of oxidative stress or ionizing radiation-which produces some biochemical and morphological effects that mimic those of telomere shortening-to induce Smurf2's expression provides additional evidence that elevation of the steady-state level of Smurf2 in senescent fibroblasts is specifically an effect of telomere attrition rather than a consequence of cellular stress.

Adventitious expression of Smurf2 at the same physiological level observed during senescence occurring secondary to telomere attrition is sufficient to produce the senescence phenotype in both early passage fibroblasts and telomerase-immortalized cells. These effects of Smurf2 are likely to be independent of any substantial telomere shortening, as they were observed after as few as four population doublings in cells that received Smurf2-expressing constructs. The reversal of hTERTmediated cellular immortalization by adventitious expression of Smurf2 and the lack of a detectable DNA damage response in cells undergoing Smurf2-mediated senescence provide further evidence that the senescenceinducing actions of Smurf2 are a consequence rather 
than cause of telomere attrition. To our knowledge, Smurf 2 is the first gene identified to be both regulated by progressive telomere shortening and sufficient to produce the senescence phenotype. We suggest that adventitious expression of Smurf 2 in early passage cells may provide a useful tool for study of the senescence phenotype in fibroblasts that have not undergone extended passage in culture or experienced cellular stress.

While activation of a response to DNA damage has been proposed as the event responsible for the telomeredependent signaling of the onset of senescence (Blackburn 2001; Karlseder et al. 2002; d'Adda di Fagagna et al. 2003; Takai et al. 2003; Herbig et al. 2004), the number of nuclear $\gamma$-H2A.X foci observed during replicative senescence is much lower than the number seen in cells exposed to ionizing radiation or expressing a dominantnegative TRF2 mutant protein (d'Adda di Fagagna et al. 2003; Takai et al. 2003; Herbig et al. 2004; also see Fig. 3). Our finding that expression of Smurf 2 is induced by telomere attrition during replicative senescence, but not by DNA-damaging agents or mutant TRF2-mediated telomere dysfunction supports the view that the DNA damage response activated by telomere attrition during replicative senescence differs from that invoked by ionizing radiation or inhibition of TRF2 (Herbig et al. 2004).

The existence of multiple senescence-related complementation groups (Pereira-Smith and Smith 1988) suggests that senescence can be mediated through parallel pathways - any one of which is sufficient to confer the senescence phenotype. This view is supported by the dramatically disparate alterations in gene expression observed during replicative senescence in human fibroblasts versus human mammary epithelial cells (HMECs) (Zhang et al. 2003, 2004). Even HMECs of the same origin cultured under different conditions can display disparate gene expression patterns when entering senescence (Zhang et al. 2004), providing further evidence that senescence is a diverse cellular process. Consistent with such observations, Smurf2 elevation was not seen during telomere-dependent senescence in either post-selection HMECs (Fig. 1A), which lose the ability to express p $16^{\text {INK4a }}$ during the process of selection from M0 arrest (Brenner et al. 1998; Huschtscha et al. 1998), or in HMECs cultured on feeder layers (Zhang et al. 2004), which do not go through the M0-selection process and maintain normal p16 ${ }^{\mathrm{INK} 4 \mathrm{a}}$ expression (Ramirez et al. 2001; Herbert et al. 2002). However, adventitious expression of Smurf2 in early passage HMECs was sufficient to induce the senescence phenotype (data not shown). Collectively, our findings support the argument that Smurf2 up-regulation mediates one of the multiple cellular pathways that have been proposed to lead to senescence (Pereira-Smith and Smith 1988). The conclusion that senescence can also occur through Smurf2-independent mechanisms was supported by experiments in which stable expression of Smurf2-specific short interfering RNAs (SiRNAs) in mid-passage BJ and WS1 fibroblasts were found not to abrogate telomere-mediated senescence despite a $50 \%$ reduction in the Smurf2 protein level (data not shown).
As a member of the CIP/KIP family of CDK inhibitors, p21 inhibits the activity of cyclin D/CDK and cyclin E/CDK complexes and negatively regulates cell cycling (Sherr and Roberts 1999). Elevation of both the p21 and cyclin D1 proteins has been reported during replicative senescence associated with telomere shortening (Dulic et al. 1993; Noda et al. 1994; Atadja et al. 1995; Tahara et al. 1995; Stein et al. 1999), and we observed similar concurrent elevation of both proteins during Smurf2-induced senescence. This increase in p21 and cyclin D1 expression appears to be specific to proliferative arrest associated with the senescence phenotype, as neither gene showed elevated expression during quiescence-induced cell growth arrest (Fig. 7A). Up-regulation of p21 during senescence is believed to be dependent on signal(s) initiated by telomere shortening, as expression of hTERT blocks this up-regulation (Modestou et al. 2001; Wei et al. 2001). Our microarray analysis showed that changes in gene expression occurring in early passage cells that adventitiously express Smurf2 largely recapitulated those seen during adventitious overexpression of p21 (Chang et al. 2000). However, p21-null mutant cells adventitiously expressing Smurf2 nevertheless were observed to enter senescence, indicating that p21 up-regulation is not essential to the senescence program. Entirely parallel results have been reported for human cells undergoing senescence induced by telomere shortening (Medcalf et al. 1996).

Replicative senescence of human cells can be prevented by suppression of both the p53 and the Rb pathways (Shay and Wright 1989; Shay et al. 1991). We found that Smurf2-induced senescence also requires that at least one of these pathways be functional. While concurrent expression of HPV16 E6 and E7 oncoproteins abrogated the senescence phenotypes associated with Smurf2 elevation, expression of either oncoprotein alone was insufficient to do so. A requirement for either p53 or Rb exists in both telomere-mediated and Smurf2-mediated senescence, supporting the idea that adventitious Smurf2 up-regulation bypasses a need for telomere attrition in senescence induction. The microarray data generated in this study identify genes that may be implicated in senescence-signaling events occurring between telomere attrition and Smurf2 up-regulation, and also genes that may function downstream of Smurf2.

Smurf2 originally was identified as an E3 ubiquitin ligase based on its sequence similarity to Smurf1, a mediator of degradation of Smads $(\operatorname{Smad} 1 / 5)$ of the bone morphogenetic protein pathway (Zhu et al. 1999). Smurf2, like Smurf1, interacts with Smad proteins (Kavsak et al. 2000; Lin et al. 2000; Bonni et al. 2001; Zhang et al. 2001), and when ectopically expressed in human embryonic kidney 293 cells, promotes ubiquitination and proteasome-dependent degradation of Smad2 (Lin et al. 2000) and Smad1 (Zhang et al. 2001). Smurf2 additionally has been shown to promote formation of Smurf2/Smad complexes that degrade TGF- $\beta$ receptors (Kavsak et al. 2000) and the corepressor of Smad transcription, SnoN (Bonni et al. 2001). However, we did not observe alteration of the steady-state level of the Smad2 
or SnoN protein in fibroblasts undergoing senescence secondary to either telomere shortening or adventitious expression of Smurf2 (data not shown); transcriptional changes in genes regulated by TGF- $\beta$ also were largely not recapitulated in either group of senescent cells (Supplementary Fig. S1). Importantly, adventitious expression in early passage cells or hTERT-immortalized cells of either wild-type Smurf2 or a Smurf2 mutant lacking the ability to function as a ubiquitin ligase resulted in proliferative arrest together with biochemical, morphological, and gene expression changes characteristic of replicative senescence-indicating that Smurf2-induced senescence is not dependent on its E3 activity. Rather, this novel function of Smurf2 depends on the intactness of three WW protein-interaction domains acting together with the HECT domain in a non-ligase-dependent manner. While the $\mathrm{N}$-terminal WW domain of Smurf2 is dispensable for interaction with and subsequent degradation of Smads, deletion of this WW domain abolishes Smurf2's ability to induce senescence, suggesting that this domain may mediate interaction with one or more proteins critical for Smurf2's senescence-inducing function.

\section{Materials and methods}

\section{Cell cultures}

Human primary fibroblast cell lines BJ (from newborn foreskin), WS1 (from fetal skin), and WI38 (from fetal lung) were purchased from ATCC. The p21 $1^{-/-}$human fibroblast cell line and its parental cell line LF1 (a gift from Dr. John Sedivy, Brown University, Providence, RI) were described previously (Brown et al. 1997). All fibroblasts and human fibrosarcoma cell line HT1080 (purchased from ATCC) were cultured in Dulbecco's Modified Eagle's medium supplemented with 10\% fetal bovine serum, $100 \mathrm{U} / \mathrm{mL}$ penicillin, and $100 \mu \mathrm{g} / \mathrm{mL}$ streptomycin (Invitrogen). Two post-selection human mammary epithelial cell lines, 48R and 184 (both from normal female breasts), were gifts from Drs. Paul Yaswen and Martha Stampfer (Lawrence Berkeley National Laboratory, Berkeley, CA) and were maintained in mammary epithelia growth medium (BioWhittaker) buffered with HEPES (Sigma) to $\mathrm{pH}$ 7.4. All cells were cultured in a humidified chamber containing $5 \% \mathrm{CO}_{2}$ at $37^{\circ} \mathrm{C}$.

Confluent fibroblasts were treated with $250 \mu \mathrm{M} \mathrm{H}_{2} \mathrm{O}_{2}$ for $2 \mathrm{~h}$ and plated at subconfluent density $48 \mathrm{~h}$ after treatment. These cells efficiently developed a senescence-like oxidative stress response, including proliferative arrest and staining positive for $\beta$-galactosidase activity at $\mathrm{pH} 6$.

Early passage proliferating BJ and WS1 fibroblasts at subconfluence density were irradiated using a ${ }^{137} \mathrm{Cs}$ radiator with different doses delivered at a rate of $\sim 10 \mathrm{~Gy} / \mathrm{min}$. Cells were then analyzed 1, 24, 48, 96, or $168 \mathrm{~h}$ after irradiation. Similarly, these cells were treated with $1 \mu \mathrm{M}$ Adriamycin (Sigma) for $2 \mathrm{~h}$ or with $2.5 \mu \mathrm{M}$ etoposide (Sigma) for $17 \mathrm{~h}$, and analyzed $24 \mathrm{~h}$ after treatment.

\section{Plasmid constructs}

A retroviral expression construct, pBabe-IRES-Puro, was modified from pBabe-Puro (Morgenstern and Land 1990) by replacing the SV40 promoter with a wild-type encephalomyocarditis virus (EMCV) internal ribosome entry site (IRES), and was used in this study to adventitiously express Smurf2 protein. A DNA fragment of full-length Smurf2 open reading frame according to the published sequence (AF301463) was amplified by RT-PCR using poly(A) RNA extracted from senescent WS1 cells, and inserted into BamHI and EcoRI sites of pBabe-IRES-Puro. A BamHI-EcoRI GFP fragment released from pEGFP-C1 (Clontech) was inserted into pBabe-IRES-Puro as control. A PCRbased approach was used to generate a ubiquitin-ligase inactive mutant of Smurf2, C716A, in which cysteine 716 (TGC) was replaced by alanine (GCC). Various portions of Smurf2, including the C2 domain (amino acids 1-147), three WW domains (amino acids 149-344), the HECT domain (amino acids 345748), N-ter (amino acids 1-344), $\Delta \mathrm{C} 2$ (amino acids 149-748), and $\Delta$ WW1 (amino acids 242-748), were generated by PCR using pBabe-Smurf2-IRES-Puro as the template. These resulting constructs use IRES to express Puro and either Smurf2 or GFP from the same transcript. Smurf2 cDNA was also cloned into pBabePuro and pBabe-hygro to express Smurf2 protein separately from the selectable markers. All constructs were verified by sequencing.

A lentiviral construct, pLenti-CMV-loxP (P. Kowalski, unpubl.), was used to express HPV16 E6 and E7 oncoproteins. The cDNA of E6 and E7 was kindly provided by Dr. Kathleen Cho (University of Michigan, Ann Arbor, MI) and inserted into the lentiviral vector after the CMV promoter. DNA fragments containing the SV40 early promoter and a gene encoding hygromycin resistance or the SV40 early promoter and a gene encoding blasticidin resistance were inserted after E6 or E7 cDNA, respectively.

The retroviral construct pBabe-hygro-hTERT containing the catalytic subunit of human telomerase hTERT (a gift from Dr. Robert Weinberg, Massachusetts Institute of Technology, Cambridge, MA) was described previously (Counter et al. 1998). The retroviral constructs pLPC-NMYCTRF2 and pLPCNMYCTRF2 ${ }^{\Delta \mathrm{B} \Delta \mathrm{M}}$ (a gift from Dr. Titia de Lange, Rockefeller University, New York) were described previously (van Steensel et al. 1998).

\section{Retrovirus and lentivirus production}

Retroviral vectors were cotransfected with a plasmid (pMD2.VSV-G) encoding vescular stomatitis virus G (VSV-G) protein into Phoenix packaging cells (from Garry Nolan, Stanford University, Stanford, CA) by the calcium phosphate transfection method. Lentiviral vectors were cotransfected with a plasmid (pMD2.VSV-G) encoding VSV-G protein and a plasmid (pCMVdR8.74) encoding packaging proteins into 293T cells (Follenzi et al. 2000). VSV-G pseudotyped virus was collected 48 $\mathrm{h}$ after transfection and used to infected target cells in the presence of $4 \mu \mathrm{g} / \mathrm{mL}$ polybrene. Two days later, infected cells were selected with $0.8 \mu \mathrm{g} / \mathrm{mL}$ puromycin (Sigma), $100 \mu \mathrm{g} / \mathrm{mL}$ Hygromycin B (Calbiochem), or $10 \mu \mathrm{g} / \mathrm{mL}$ blasticidin (Invitrogen).

\section{Cell staining and growth curves}

After Smurf2 retroviral infection and puromycin selection, puromycin-resistant cells were washed with PBS twice, then stained with $1 \%$ crystal violet (Sigma) in 30\% ethanol for $5 \mathrm{~min}$. Staining for $\beta$-galactosidase activity at $\mathrm{pH} 6$ was carried out as described (Dimri et al. 1995). For growth curves, puromycinresistant cells were trypsinized and plated at low density ( 2 or $3 \times 10^{4}$ cells per well) in six-well plates. These cells were trypsinized and counted using a Coulter counter (Beckman) in triplicates every day for a week.

\section{Northern and Western analysis}

Poly(A) RNA was extracted using a FastTrack 2.0 kit (Invitrogen), and $1 \mu \mathrm{g}$ of RNA was separated on agarose gels. Hybrid- 
ization was performed using ${ }^{32} \mathrm{P}$-labeled probes generated by random priming of Smurf2 cDNA fragments.

Whole-cell lysates were collected using RIPA buffer $(50 \mathrm{mM}$ Tris- $\mathrm{HCl}$ at $\mathrm{pH} 7.5,150 \mathrm{mM} \mathrm{NaCl}, 1 \%$ Triton $\mathrm{X}-100,0.1 \%$ SDS, $0.5 \%$ deoxycholic acid, $0.02 \%$ sodium azide) with freshly added complete protease inhibitors (Roche). Protein lysates $(20 \mu \mathrm{g})$ were separated by SDS-PAGE and transferred to nitrocellulose membranes (Osmonics). Immunoblots were analyzed by Western blotting and visualized by Western lightening chemiluminescence detection (PerkinElmer). Primary antibodies used in this study were Smurf2 (Upstate Biotechnology), p21 (Ab-1, Calbiochem), p53 (DO-1, Santa Cruz Biotechnology), cyclin D1 (DCS-6, Pharmingen), Rb (4H1, Cell signaling), p27 (G173-524, Pharmingen), p16 (Upstate Biotechnology), p14 (Ab-2, NeoMarkers), c-Myc (9E10, Sigma), HPV16-E7 (sc-6981, Santa Cruz Biotechnology), and $\alpha$-tubulin (DM1A, Sigma).

\section{Immunofluorescence staining}

Cells grown on coverslips were fixed with $95 \%$ ethanol and $5 \%$ acetic acid. After blocking with 3\% BSA in PBS, cells were incubated with anti-phospho-histone H2A.X (Ser 139) antibody (JBW301, Upstate Biotechnology). Texas Red-conjugated goat anti-mouse IgG (Molecular Probes) was used as secondary antibody. DNA was also stained with DAPI. Cells were then analyzed using a Leica TCS SP2 confocal microscope.

\section{Microarray analysis}

cDNA microarrays containing 41,805 IMAGE clones representing 29,043 unique genes were obtained from the Stanford Functional Genomic Facility. Three early passage primary human fibroblast cell lines (BJ, WS1, and WI38) were infected with retrovirus made with pBabe-GFP-IRES-Puro, pBabe-Smurf2IRES-Puro and pBabe-C716A-IRES-Puro. After selection with puromycin (Sigma) for a week, poly(A) RNA was extracted using a FastTrack 2.0 kit (Invitrogen) and labeled by reverse transcription using Superscript II enzyme (Invitrogen) and oligo(dT) ${ }_{18}$ primer (New England BioLabs) in the presence of Cy3-dUTP or Cy5-dUTP (Amersham). Microarray hybridization, data collection, data normalization, and data selection followed previously described procedures (Zhang et al. 2003). Raw data were deposited into the Stanford Microarray Database (http://smd. stanford.edu). Data were then analyzed using GABRIEL (http:// gabriel.stanford.edu; Pan et al. 2002) with two criteria applied: a $\mathrm{t}$-score threshold of 2 to give a $\mathrm{p}$-value cutoff of 0.05 and average expression threshold of 1.5 .

\section{Acknowledgments}

We thank Drs. P. Kowalski, T. de Lange, J. Sedivy, M. Stampfer, P. Yaswen, G. Nolan, and R. Weinberg for kindly providing reagents; Y. Teng and R. Lin for excellent technical assistance; and members of the Cohen lab for helpful discussions and assistance. This work was supported by Postdoctoral Fellowship 5FB-0067 from the California Breast Cancer Research Program to H.Z. and by grants from the National Foundation for Cancer Research and The Ellison Medical Foundation to S.N.C.

\section{References}

Atadja, P., Wong, H., Veillete, C., and Riabowol, K. 1995. Overexpression of cyclin D1 blocks proliferation of normal diploid fibroblasts. Exp. Cell Res. 217: 205-216.

Blackburn, E.H. 2001. Switching and signaling at the telomere.
Cell 106: 661-673.

Bodnar, A.G., Ouellette, M., Frolkis, M., Holt, S.E., Chiu, C.P., Morin, G.B., Harley, C.B., Shay, J.W., Lichtsteiner, S., and Wright, W.E. 1998. Extension of life-span by introduction of telomerase into normal human cells. Science 279: 349-352.

Bonni, S., Wang, H.R., Causing, C.G., Kavsak, P., Stroschein, S.L., Luo, K., and Wrana, J.L. 2001. TGF- $\beta$ induces assembly of a Smad2-Smurf2 ubiquitin ligase complex that targets SnoN for degradation. Nat. Cell Biol. 3: 587-595.

Brenner, A.J., Stampfer, M.R., and Aldaz, C.M. 1998. Increased p16 expression with first senescence arrest in human mammary epithelial cells and extended growth capacity with p16 inactivation. Oncogene 17: 199-205.

Brown, J.P., Wei, W., and Sedivy, J.M. 1997. Bypass of senescence after disruption of $\mathrm{p} 21^{\mathrm{CIP} 1 / \mathrm{WAF} 1}$ gene in normal diploid human fibroblasts. Science 277: 831-834.

Chang, B.D., Watanabe, K., Broude, E.V., Fang, J., Poole, J.C., Kalinichenko, T.V., and Roninson, I.B. 2000. Effects of p21Waf1/Cip1/Sdi1 on cellular gene expression: Implications for carcinogenesis, senescence, and age-related diseases. Proc. Natl. Acad. Sci. 97: 4291-4296.

Chen, Q., Fischer, A., Reagan, J.D., Yan, L.J., and Ames, B.N. 1995. Oxidative DNA damage and senescence of human diploid fibroblast cells. Proc. Nat1. Acad. Sci. 92: 4337-4341.

Counter, C.M., Hahn, W.C., Wei, W., Caddle, S.D., Beijersbergen, R.L., Lansdorp, P.M., Sedivy, J.M., and Weinberg, R.A. 1998. Dissociation among in vitro telomerase activity, telomere maintenance, and cellular immortalization. Proc. Natl. Acad. Sci. 95: 14723-14728.

d'Adda di Fagagna, F., Reaper, P.M., Clay-Farrace, L., Fiegler, H., Carr, P., Von Zglinicki, T., Saretzki, G., Carter, N.P., and Jackson, S.P. 2003. A DNA damage checkpoint response in telomere-initiated senescence. Nature 426: 194-198.

de Lange, T. 2002. Protection of mammalian telomeres. Oncogene 21: 532-540.

Di Leonardo, A., Linke, S.P., Clarkin, K., and Wahl, G.M. 1994. DNA damage triggers a prolonged p53-dependent G1 arrest and long-term induction of Cip1 in normal human fibroblasts. Genes \& Dev. 8: 2540-2551.

Dickson, M.A., Hahn, W.C., Ino, Y., Ronfard, V., Wu, J.Y., Weinberg, R.A., Louis, D.N., Li, F.P., and Rheinwald, J.G. 2000. Human keratinocytes that express hTERT and also bypass a

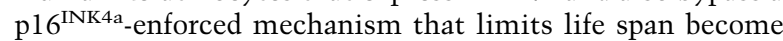
immortal yet retain normal growth and differentiation characteristics. Mol. Cell. Biol. 20: 1436-1447.

Dimri, G.P., Lee, X., Basile, G., Acosta, M., Scott, G., Roskelley, C., Medrano, E.E., Linskens, M., Rubelj, I., Pereira-Smith, O., et al. 1995. A biomarker that identifies senescent human cells in culture and in aging skin in vivo. Proc. Natl. Acad. Sci. 92: 9363-9367.

Dulic, V., Drullinger, L.F., Lees, E., Reed, S.I., and Stein, G.H. 1993. Altered regulation of G1 cyclins in senescent human diploid fibroblasts: Accumulation of inactive cyclin E-Cdk2 and cyclin D1-Cdk2 complexes. Proc. Nat1. Acad. Sci. 90: $11034-11038$.

Follenzi, A., Ailles, L.E., Bakovic, S., Geuna, M., and Naldini, L. 2000. Gene transfer by lentiviral vectors is limited by nuclear translocation and rescued by HIV-1 pol sequences. Nat. Genet. 25: 217-222.

Gorbunova, V., Seluanov, A., and Pereira-Smith, O.M. 2002. Expression of human telomerase (hTERT) does not prevent stress-induced senescence in normal human fibroblasts but protects the cells from stress-induced apoptosis and necrosis. J. Biol. Chem. 277: 38540-38549.

Harley, C.B. 1991. Telomere loss: Mitotic clock or genetic time bomb? Mutat. Res. 256: 271-282. 
Hayflick, L. 1976. The cell biology of human aging. N. Engl. J. Med. 295: 1302-1308.

Hayflick, L. and Moorhead, P.S. 1961. The serial cultivation of human diploid cell strains. Exp. Cell Res. 25: 585-621.

Herbert, B.S., Wright, W.E., and Shay, J.W. 2002. p16 ${ }^{\text {INK4a }}$ inactivation is not required to immortalize human mammary epithelial cells. Oncogene 21: 7897-7900.

Herbig, U., Jobling, W.A., Chen, B.P., Chen, D.J., and Sedivy, J.M. 2004. Telomere shortening triggers senescence of human cells through a pathway involving ATM, p53, and p21 ${ }^{\mathrm{CIP} 1}$, but not p16 $6^{\mathrm{INK} 4 \mathrm{a}}$. Mol. Cell 14: 501-513.

Holt, S.E., Shay, J.W., and Wright, W.E. 1996. Refining the telomere-telomerase hypothesis of aging and cancer. Nat. Biotechnol. 14: 836-839.

Huschtscha, L.I., Noble, J.R., Neumann, A.A., Moy, E.L., Barry, P., Melki, J.R., Clark, S.J., and Reddel, R.R. 1998. Loss of p16INK4 expression by methylation is associated with lifespan extension of human mammary epithelial cells. Cancer Res. 58: 3508-3512.

Kang, Y., Chen, C.R., and Massague, J. 2003. A self-enabling TGF $\beta$ response coupled to stress signaling: Smad engages stress response factor ATF3 for Id1 repression in epithelial cells. Mol. Cell 11: 915-926.

Karlseder, J., Smogorzewska, A., and de Lange, T. 2002. Senescence induced by altered telomere state, not telomere loss. Science 295: 2446-2449.

Kavsak, P., Rasmussen, R.K., Causing, C.G., Bonni, S., Zhu, H., Thomsen, G.H., and Wrana, J.L. 2000. Smad7 binds to Smurf2 to form an E3 ubiquitin ligase that targets the TGF $\beta$ receptor for degradation. Mol. Cell 6: 1365-1375.

Kim, N.W., Piatyszek, M.A., Prowse, K.R., Harley, C.B., West, M.D., Ho, P.L., Coviello, G.M., Wright, W.E., Weinrich, S.L., and Shay, J.W. 1994. Specific association of human telomerase activity with immortal cells and cancer. Science 266: 2011-2015.

Kim, S.H., Kaminker, P., and Campisi, J. 2002. Telomeres, aging and cancer: In search of a happy ending. Oncogene 21: 503511.

Kiyono, T., Foster, S.A., Koop, J.I., McDougall, J.K., Galloway, D.A., and Klingelhutz, A.J. 1998. Both Rb/p16INK4a inactivation and telomerase activity are required to immortalize human epithelial cells. Nature 396: 84-88.

Lamb, J., Ramaswamy, S., Ford, H.L., Contreras, B., Martinez, R.V., Kittrell, F.S., Zahnow, C.A., Patterson, N., Golub, T.R., and Ewen, M.E. 2003. A mechanism of cyclin D1 action encoded in the patterns of gene expression in human cancer. Cell 114: 323-334.

Lin, X., Liang, M., and Feng, X.H. 2000. Smurf2 is a ubiquitin E3 ligase mediating proteasome-dependent degradation of Smad2 in transforming growth factor- $\beta$ signaling. J. Biol. Chem. 275: 36818-36822.

Massague, J. 2000. How cells read TGF- $\beta$ signals. Nat. Rev. Mol. Cell Biol. 1: 169-178.

Medcalf, A.S., Klein-Szanto, A.J., and Cristofalo, V.J. 1996. Expression of $\mathrm{p} 21$ is not required for senescence of human fibroblasts. Cancer Res. 56: 4582-4585.

Modestou, M., Puig-Antich, V., Korgaonkar, C., Eapen, A., and Quelle, D.E. 2001. The alternative reading frame tumor suppressor inhibits growth through p21-dependent and p21-independent pathways. Cancer Res. 61: 3145-3150.

Morgenstern, J.P. and Land, H. 1990. Advanced mammalian gene transfer: High titre retroviral vectors with multiple drug selection markers and a complementary helper-free packaging cell line. Nucleic Acids Res. 18: 3587-3596.

Munger, K. and Howley, P.M. 2002. Human papillomavirus im- mortalization and transformation functions. Virus Res. 89: $213-228$.

Noda, A., Ning, Y., Venable, S.F., Pereira-Smith, O.M., and Smith, J.R. 1994. Cloning of senescent cell-derived inhibitors of DNA synthesis using an expression screen. Exp. Cell Res. 211: 90-98.

Pan, K.H., Lih, C.J., and Cohen, S.N. 2002. Analysis of DNA microarrays using algorithms that employ rule-based expert knowledge. Proc. Natl. Acad. Sci. 99: 2118-2123.

Pereira-Smith, O.M. and Smith, J.R. 1988. Genetic analysis of indefinite division in human cells: Identification of four complementation groups. Proc. Natl. Acad. Sci. 85: 60426046.

Ramirez, R.D., Morales, C.P., Herbert, B.S., Rohde, J.M., Passons, C., Shay, J.W., and Wright, W.E. 2001. Putative telomere-independent mechanisms of replicative aging reflect inadequate growth conditions. Genes \& Dev. 15: 398-403.

Robles, S.J. and Adami, G.R. 1998. Agents that cause DNA double strand breaks lead to $\mathrm{p} 16^{\mathrm{INK} 4 \mathrm{a}}$ enrichment and the premature senescence of normal fibroblasts. Oncogene 16: $1113-1123$.

Romanov, S.R., Kozakiewicz, B.K., Holst, C.R., Stampfer, M.R., Haupt, L.M., and Tisty, T.D. 2001. Normal human mammary epithelial cells spontaneously escape senescence and acquire genomic changes. Nature 409: 633-637.

Serrano, M., Lin, A.W., McCurrach, M.E., Beach, D., and Lowe, S.W. 1997. Oncogenic ras provokes premature cell senescence associated with accumulation of p53 and p16 $6^{\mathrm{INK} 4 \mathrm{a}}$. Cell 88: 593-602.

Severino, J., Allen, R.G., Balin, S., Balin, A., and Cristofalo, V.J. 2000. Is $\beta$-galactosidase staining a marker of senescence in vitro and in vivo? Exp. Cell Res. 257: 162-171.

Shay, J.W. and Wright, W.E. 1989. Quantitation of the frequency of immortalization of normal human diploid fibroblasts by SV40 large T-antigen. Exp. Cell Res. 184: 109-118.

Shay, J.W., Pereira-Smith, O.M., and Wright, W.E. 1991. A role for both RB and p53 in the regulation of human cellular senescence. Exp. Cell Res. 196: 33-39.

Sherr, C.J. 1994. G1 phase progression: Cycling on cue. Cell 79: $551-555$.

Sherr, C.J. and Roberts, J.M. 1999. CDK inhibitors: Positive and negative regulators of G1-phase progression. Genes \& Dev. 13: 1501-1512.

Smogorzewska, A. and de Lange, T. 2002. Different telomere damage signaling pathways in human and mouse cells. EMBO J. 21: 4338-4348.

Stein, G.H., Drullinger, L.F., Soulard, A., and Dulic, V. 1999. Differential roles for cyclin-dependent kinase inhibitors p21 and p16 in the mechanisms of senescence and differentiation in human fibroblasts. Mol. Cell. Biol. 19: 2109-2117.

Stewart, S.A., Ben-Porath, I., Carey, V.J., O'Connor, B.F., Hahn, W.C., and Weinberg, R.A. 2003. Erosion of the telomeric single-strand overhang at replicative senescence. Nat. Genet. 33: 492-496.

Tahara, H., Kamada, K., Sato, E., Tsuyama, N., Kim, J.K., Hara, E., Oda, K., and Ide, T. 1995. Increase in expression levels of interferon-inducible genes in senescent human diploid fibroblasts and in SV40-transformed human fibroblasts with extended lifespan. Oncogene 11: 1125-1132.

Takai, H., Smogorzewska, A., and de Lange, T. 2003. DNA damage foci at dysfunctional telomeres. Curr. Biol. 13: 15491556.

van Steensel, B., Smogorzewska, A., and de Lange, T. 1998. TRF2 protects human telomeres from end-to-end fusions. Cell 92: 401-413. 
Vaziri, H. and Benchimol, S. 1998. Reconstitution of telomerase activity in normal human cells leads to elongation of telomeres and extended replicative life span. Curr. Biol. 8: 279282.

von Zglinicki, T., Saretzki, G., Docke, W., and Lotze, C. 1995. Mild hyperoxia shortens telomeres and inhibits proliferation of fibroblasts: A model for senescence? Exp. Cell Res. 220: 186-193.

Wei, S. and Sedivy, J.M. 1999. Expression of catalytically active telomerase does not prevent premature senescence caused by overexpression of oncogenic Ha-Ras in normal human fibroblasts. Cancer Res. 59: 1539-1543.

Wei, W., Hemmer, R.M., and Sedivy, J.M. 2001. Role of p14 ${ }^{\mathrm{ARF}}$ in replicative and induced senescence of human fibroblasts. Mol. Cell. Biol. 21: 6748-6757.

Wright, W.E. and Shay, J.W. 1992. Telomere positional effects and the regulation of cellular senescence. Trends Genet. 8: 193-197.

- 2001. Cellular senescence as a tumor-protection mechanism: The essential role of counting. Curr. Opin. Genet. Dev. 11: 98-103.

. 2002. Historical claims and current interpretations of replicative aging. Nat. Biotechnol. 20: 682-688.

Zhang, Y., Chang, C., Gehling, D.J., Hemmati-Brivanlou, A., and Derynck, R. 2001. Regulation of Smad degradation and activity by Smurf2, an E3 ubiquitin ligase. Proc. Nat1. Acad. Sci. 98: 974-979.

Zhang, H., Pan, K.H., and Cohen, S.N. 2003. Senescence-specific gene expression fingerprints reveal cell-type-dependent physical clustering of up-regulated chromosomal loci. Proc. Natl. Acad. Sci. 100: 3251-3256.

Zhang, H., Herbert, B.S., Pan, K.H., Shay, J.W., and Cohen, S.N. 2004. Disparate effects of telomere attrition on gene expression during replicative senescence of human mammary epithelial cells cultured under different conditions. Oncogene 23: 6193-6198.

Zhu, H., Kavsak, P., Abdollah, S., Wrana, J.L., and Thomsen, G.H. 1999. A SMAD ubiquitin ligase targets the BMP pathway and affects embryonic pattern formation. Nature 400: $687-693$. 


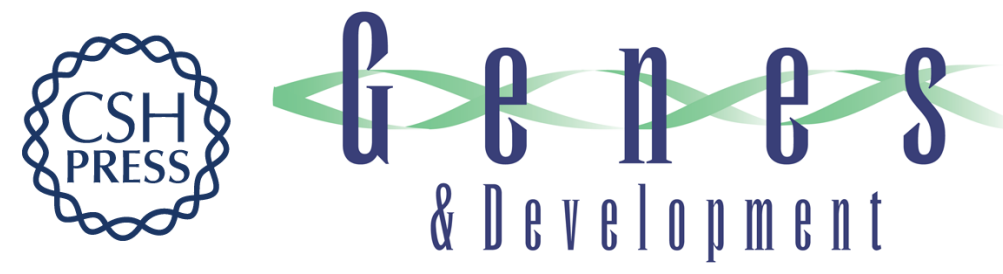

\section{Smurf2 up-regulation activates telomere-dependent senescence}

Hong Zhang and Stanley N. Cohen

Genes Dev. 2004, 18:

Access the most recent version at doi:10.1101/gad.1253004

Supplemental

Material

References

This article cites 65 articles, 26 of which can be accessed free at:

http://genesdev.cshlp.org/content/18/24/3028.full.html\#ref-list-1

\section{License}

Email Alerting Service

http://genesdev.cshlp.org/content/suppl/2004/12/01/gad.1253004.DC1 right corner of the article or click here.

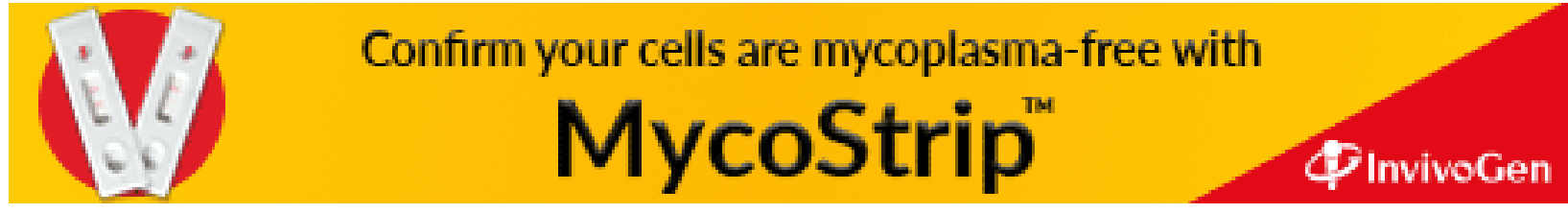

